\title{
THORACIC DUCT INJURY AFTER MASTECTOMY - CASE REPORT AND LITERATURE REVIEW
}

\author{
Juliana Ferreira de Lima¹, Bruna Anderson¹, Antônio Carlos Toshihiro Nisida¹, Fabiano Cataldi Engel¹, Luiz \\ Henrique Gebrim¹ \\ ${ }^{1}$ Hospital Pérola Byington - São Paulo (SP), Brazil
}

Introduction: In adults, the thoracic duct carries about four liters of lymph per day, and its injury can lead to rapid accumulation in the pleural cavity, causing acute and chronic changes in lung dynamics. Thoracic duct injuries are uncommon and occur during surgical procedures in the thoracic region and trauma, such as esophagectomy, mediastinal and pleuro-pulmonary surgical procedures. Surgical injuries have been described after almost all types of thoracic surgical procedures, especially those performed in the upper left thoracic strait. The clinical diagnosis is based most often on the high output of the introduced chest drain, accompanied by a milky aspect. In addition, computed tomography, lymphoscintigraphy and lymphanangiography are possible methods for diagnosis. Clinical series on duct injuries after thoracic surgery report that in approximately $25 \%$ to $50 \%$ of cases spontaneous closure of the fistula occurs with conservative measures, after the introduction of parenteral nutrition or enteral diet with medium chain triglycerides. The other cases needed clinical and surgical treatment. Operative treatment consists of performing videothoracoscopy or right thoracotomy with identification of the lymphatic duct, followed by ligation. Case report: A 48-year-old woman, born in Santana do Parnaíba, state of São Paulo, with a diagnosis of breast cancer on the left, histological type of invasive breast carcinoma Luminal B, who underwent a modified radical mastectomy (Madden technique) on the left with immediate breast reconstruction. In the intraoperative period, important involvement of the left axillary lymph nodes was evidenced, which may have distorted lymphatic vessels and ducts. It evolved in the late postoperative period with high milky drainage in a suction drain in the left axillary region. Then, a hypothesis of thoracic duct injury was raised as a post-surgical complication. Biochemical analysis of milky secretion showed a high concentration of triglycerides and cytology describes the presence of proteinaceous material and macrophages. A conservative approach was adopted with adjustment of a hyperproteic, hypoglycidic and rich in medium chain triglyceride parenteral diet. The patient underwent lymphoscintigraphy, twenty days after the diagnostic hypothesis, but the fistula was not detected. Progressed with a decrease in the flow gradually until the drain was removed and she was discharged from the hospital in good condition. At the moment, the patient is undergoing adjuvant treatment for breast cancer. 